\title{
The Use of Percutaneous Coronary Intervention (PCI) for Heart Attack A Critique
}

\author{
Victor Gurewich* \\ Department of Medicine, Harvard Medical School, Mount Auburn Hospital, USA
}

*Corresponding author: Victor Gurewich, Department of Medicine, Harvard Med-

ical School, Mount Auburn Hospital, USA.

Received Date: September 08, 2021

Published Date: September 22, 2021

\section{Mini Review}

PCI has recently become the treatment of choice for acute myocardial infarction (AMI), an emergency procedure in which time to reperfusion of the ischemic myocardium should be as short as possible because the ischemic damage rapidly becomes irreversible. Therefore, the choice of PCI for AMI is difficult to understand, since PCI is a complicated, time-consuming hospital procedure, whereas fibrinolysis, the alternative, which PCI replaced, is so much simpler and faster. PCI requires an arterial catheterization in a hospital which takes time and is costly, whereas fibrinolysis requires only an intravenous injection or infusion, that is simple, fast and low cost. In contrast to PCI, fibrinolysis also perfuses all size vessels and is not limited to arteries larger than the size of the catheter. In view of this disparity between the two treatments, how did a timeconsuming procedure like PCI succeed to show that it was more effective than a rapidly delivered treatment like fibrinolysis for an emergency like AMI where the time to reperfusion is the ultimate criterion of success.

One large difference between these two treatments is that PCI, being an invasive treatment, is well reimbursed, up to $\$ 15,000$ per procedure, which helps to explain its popularity. This financial support comes from third party payers, and there is nothing equivalent for treatment by fibrinolysis. This reimbursement cannot help but contribute to the recent success of PCI, it was also necessary that PCI establish it superior efficacy and comparative trials between PCI and fibrinolysis were done in which PCI showed that it was slightly more effective. The problem with these comparative trials was in the way that fibrinolysis was induced. Unfortunately, fibrinolysis has not been standardized that fibrinolysis by either of the two activators or both can be called "fibrinolysis." Fibrinolysis is a natural defense against blood clots, and both of the plasminogen activators IPA and uPA, that determine its efficacy are present in normal blood. tPA starts fibrinolysis when it activates the first fibrin-bound plasminogen on the fibrin clot which is located near tPA's fibrin binding site. The initiation of fibrin degradation exposes two additional plasminogen binding sites, and their respective plasminogens are activated by uPA, both prouPA its activated form, two-chain urokinase (tcuPA).

Full or complete fibrinolysis requires both tPA and uPA []. Since their modes of action are complementary, their combined effect is synergistic [] so that the combination is more potent than the sum of the two parts, and among the two parts, tPA is responsible for one third of fibrinolysis and uPA two thirds. So tPA fibrinolysis is the least effective. In all of the comparative studies of PCI against fibrinolysis, the fibrinolysis was always fibrinolysis by tPA alone, the least effective. Comparative studies of PCI against fully effective fibrinolysis have been avoided and are unlikely to be done since PCI will lose such a test, since fibrinolysis is more than $3 \mathrm{X}$ more effective in the synergistic combination. When prouPA was discovered in 1984 [], the sequential combination of tPA and prouPA reduced the tPA requirement from $100 \mathrm{mg}$ to a $5 \mathrm{mg}$ bolus which was followed by a prouPA infusion of $40 \mathrm{mg} / \mathrm{h}$ for 90 minutes. This increased efficacy substantially from what it was with tPA alone, and due to this change, the bleeding risk was virtually eliminated. Therefore, full fibrinolysis requires both tPA and UPA in a sequential combination. Anything less than that, like tPA alone, is not fibrinolysis. Recently, to justify the use of PCI for the treatment 
of AMI, PCI was compared with "fibrinolysis" by tPA alone which is not fibrinolysis, it is a small fraction $(<1 / 3)$ of fibrinolysis. Until PCI is shown more effective than full fibrinolysis, its use cannot be justified especially since its use delays the time to reperfusion substantially.

Since time to reperfusion is the most important measure of treatment success, the delay caused by the use of PCI for the treatment of AMI is a serious liability of this treatment. PCI has never been shown be as effective as full fibrinolysis. Until this is rectified, the PCI patients are getting substandard treatment. At the very least, they deserve that their treatment be compared with complete fibrinolysis (tPA \& UPA) in AMI.

\section{Acknowledgement}

None.

\section{Conflict of Interest}

No conflict of interest. 